\title{
MODIS INSTRUMENT OPERATION AND CALIBRATION IMPROVEMENTS
}

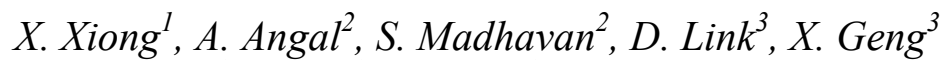 \\ B. Wenny ${ }^{3}, A$. Wu ${ }^{3}, H$. Chen ${ }^{3}$, and V. Salomonson ${ }^{4}$ \\ ${ }^{1}$ Sciences and Exploration Directorate, NASA/GSFC, Greenbelt, MD 20771, USA \\ ${ }^{2}$ Science Systems and Applications Inc., 10210 Greenbelt Road, Lanham, MD 20706, USA \\ ${ }^{3}$ Sigma Space Corporation, 4801 Forbes Boulevard, Lanham, MD 20706, USA \\ ${ }^{4}$ University of Utah, Salt Lake City, UT 84112, USA
}

\begin{abstract}
Terra and Aqua MODIS have successfully operated for over 14 and 12 years since their respective launches in 1999 and 2002. The MODIS on-orbit calibration is performed using a set of on-board calibrators, which include a solar diffuser for calibrating the reflective solar bands (RSB) and a blackbody for the thermal emissive bands (TEB). On-orbit changes in the sensor responses as well as key performance parameters are monitored using the measurements of these on-board calibrators. This paper provides an overview of MODIS onorbit operation and calibration activities, and instrument long-term performance. It presents a brief summary of the calibration enhancements made in the latest MODIS data collection 6 (C6). Future improvements in the MODIS calibration and their potential applications to the S-NPP VIIRS are also discussed.
\end{abstract}

Index Terms - MODIS, Terra, Aqua, Calibration

\section{INTRODUCTION}

Both Terra and Aqua MODIS have successfully operated for more than a decade since launch in 1999 and 2002, respectively. High quality MODIS data products have been continuously generated and widely distributed from complementary morning and afternoon observations and have significantly contributed to the study of the earth's systems and key associated environmental parameters [1]. The success of the MODIS mission is not possible without the dedicated and diligent effort made by the MODIS Characterization Support Team (MCST) to continuously operate, monitor, and calibrate both instruments [2].

MODIS has 36 spectral bands: 20 reflective solar bands (RSB) covering wavelengths from 0.41 to $2.3 \mu \mathrm{m}$ and 16 thermal emissive bands (TEB) from 3.7 to $14.5 \mu \mathrm{m}$. Its onorbit calibration and characterization are facilitated by a set of on-board calibrators (OBC), which include a solar diffuser (SD), a solar diffuser stability monitor (SDSM), a blackbody (BB), and a spectroradiometric calibration assembly (SRCA). In addition to OBC, lunar observations and data collected over selected ground targets are used to improve and maintain sensor calibration quality. This paper provides an overview of MODIS instrument operations and calibration activities. It illustrates instrument and OBC longterm performance and discusses calibration challenges to be addressed for future improvements.

\section{INSTRUMENT OPERATION AND CALIBRATION}

The MODIS Instrument Operations Team (IOT), a key component of MCST that closely interacts with the mission flight operations team (FOT), is primarily responsible for scheduling and implementing various calibration activities. Key on-orbit calibration activities include regular SD and SDSM operations, periodic BB temperature warm-up and cool-down (WUCD), electronic calibration (Ecal), and SRCA operations in different modes: radiometric, spatial, and spectral. Other calibration activities, such as nearmonthly lunar observations, are also planned and executed. Table 1 is a summary of MODIS on-orbit calibration activities performed up to May 2014. As both Terra and Aqua MODIS have operated significantly beyond their specified lifetime (6 years), some of the calibration frequencies have been gradually reduced. This applies mainly for the calibrations involving components with limited lifetimes.

Table 1 Summary of MODIS on-orbit calibration activities (up to May 1, 2014)

\begin{tabular}{|c|c|c|c|c|c|}
\hline & SD/SDSM & BB & SRCA & ECAL & Moon \\
\hline Terra & 673 & 90 & 315 & 71 & 136 \\
\hline Aqua & 518 & 52 & 249 & 60 & 111 \\
\hline
\end{tabular}

MODIS RSB calibration is referenced to the on-board SD with its on-orbit degradation tracked by the SDSM. RSB calibration coefficients, such as detector gains and responses versus scan angle (RVS), are derived and updated using detector responses to the SD, Moon, and ground targets at different angles of incidence (AOI). The TEB calibration is on a scan-by-scan basis with reference to the on-board BB. 
The SRCA is primarily used for the sensor spectral and spatial characterization [3]. Operationally, all the MODIS components and sub-systems continue to perform their designed functions.

\section{ON-ORBIT PERFORMANCE}

Both MODIS instruments continue to operate normally with a very small increase of the instrument (including scan cavity and mirror) temperatures over the mission lifetime, less than $3.5 \mathrm{~K}$ and $2.5 \mathrm{~K}$ for Terra and Aqua MODIS, respectively. The VIS and NIR focal plane assemblies (FPA) are operated without temperature control, showing similar performance. The SMIR and LWIR FPA are nominally controlled at $83 \mathrm{~K}$ and their on-orbit performance is demonstrated in Figures 1 and 2. In general, the SMIR and LWIR FPA in Terra MODIS are well-controlled with excellent stability. Due to a loss of Aqua MODIS radiative cooler margin, its SMIR and LWIR FPA (weekly granule averaged temperatures) are experiencing gradually increased orbital and seasonal variations (up to 0.2 and $0.6 \mathrm{~K}$ ) in recent years. The BB temperature is nominally controlled at $290 \mathrm{~K}$ for Terra (Figure 3) and $285 \mathrm{~K}$ for Aqua MODIS (Figure 4). In addition to very small seasonal variations ( \pm 10 $\mathrm{mK}$ ), the long-term drift of Terra BB temperatures is less than $40 \mathrm{mK}$ over a period of 14 years. For Aqua MODIS, the BB temperatures are extremely stable with little drift and variations. On-orbit degradation of the SD is monitored via regularly scheduled SDSM observations covering the wavelength range from 0.41 to $0.94 \mu \mathrm{m}$. As shown in Figure 5, both Terra and Aqua MODIS SD have experienced the same wavelength dependent degradation (larger degradation at shorter wavelengths). Due to a SD operation anomaly, the Terra MODIS SD door has been fixed at an open position since mid-2003. This configuration results in more frequent solar exposure of the SD and thus much faster SD degradation. Similar wavelength dependent SD degradation is also seen in S-NPP VIIRS [4].

Table 2 Status summary of MODIS noisy and inoperable detectors (up to May 1, 2014)

\begin{tabular}{|l|c|c|c|c|}
\hline & \multicolumn{2}{|c|}{ Terra MODIS } & \multicolumn{2}{c|}{ Aqua MODIS } \\
\hline Date / Type & Noisy & Inoperable & Noisy & Inoperable \\
\hline Pre-launch & 30 & 0 & 3 & 10 \\
\hline Nadir Door Open & 35 & 0 & 7 & 15 \\
\hline Current & 47 & 0 & 7 & 15 \\
\hline
\end{tabular}

There are a total of 490 individual detectors among the 36 MODIS spectral bands. The majority of the detectors continue to operate and function normally, meeting performance requirements in terms of SNR and NEdT. Most noisy or inoperable detectors were either identified prelaunch or on-orbit at nadir door open. Table 2 provides a status summary of the noisy and inoperable detectors for both instruments. As seen from the table, Terra MODIS has no inoperable detectors, and no new inoperable detectors have been identified for Aqua MODIS since its launch.

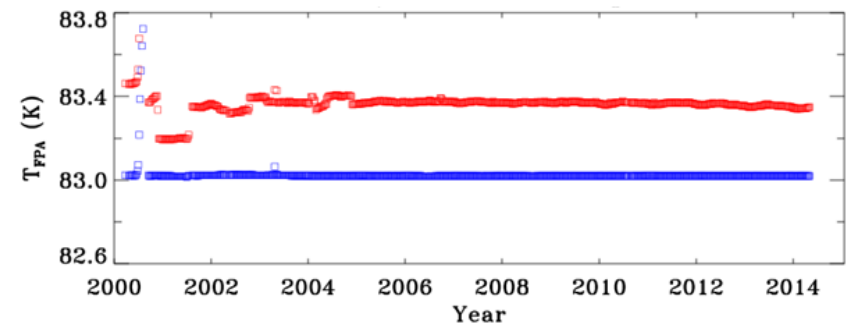

Figure 1 Terra MODIS SMIR (red) and LWIR (blue) FPA temperatures

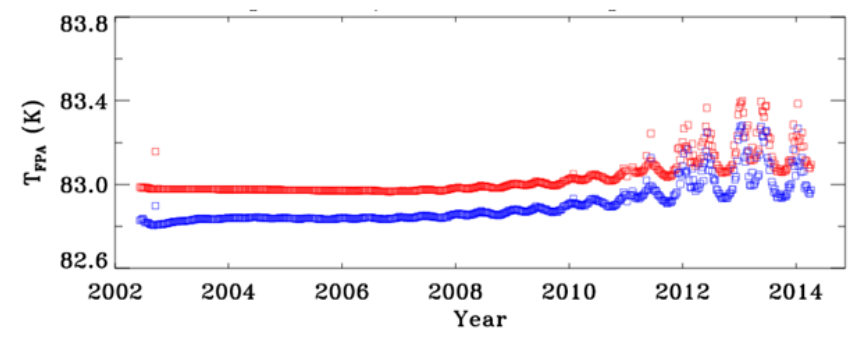

Figure 2 Aqua MODIS SMIR (red) and LWIR (blue) FPA temperatures

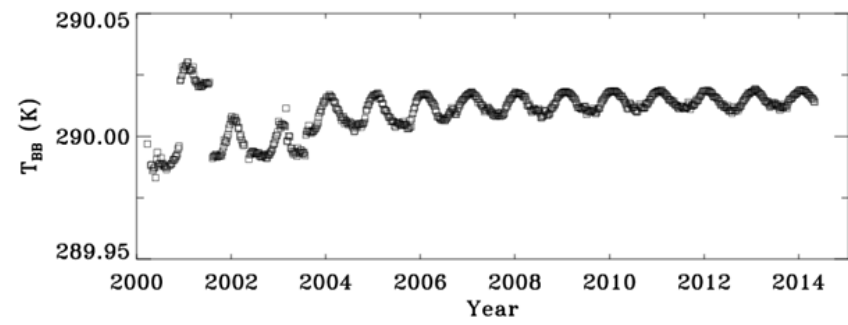

Figure 3 Terra MODIS on-board BB temperatures

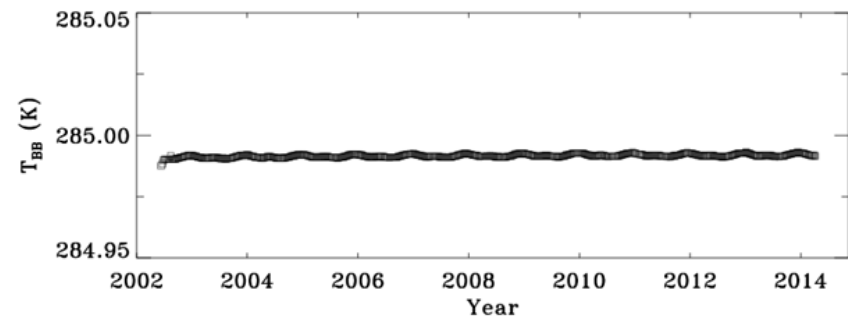

Figure 4 Aqua MODIS on-board BB temperatures

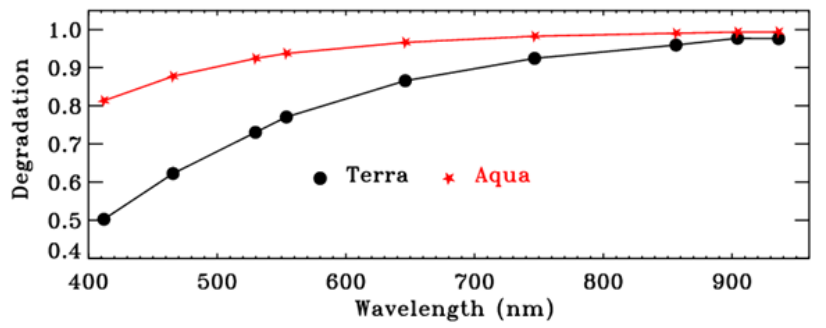

Figure 5 SD on-orbit degradation: Terra MODIS (black) and Aqua MODIS (red) 
The MODIS RSB calibration coefficients (m1) are inversely proportional to the detector responses (gains). The long-term radiometric responses of the VIS spectral bands generated from the on-board SD/SDSM calibrators are presented in Figures 6 (Terra) and 7 (Aqua). For both instruments, the bands with shorter wavelengths have shown larger gain changes.

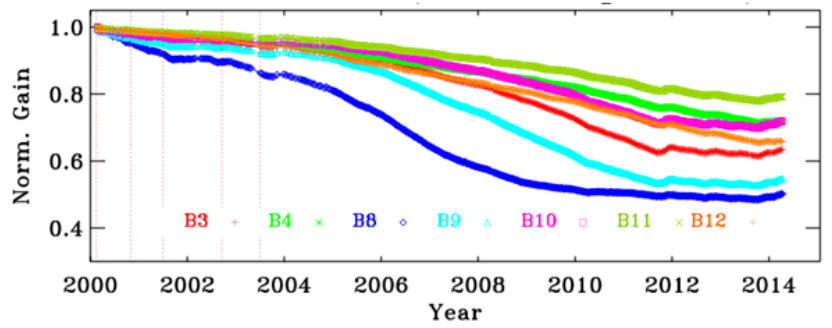

Figure 6 Normalized gains of Terra MODIS VIS bands

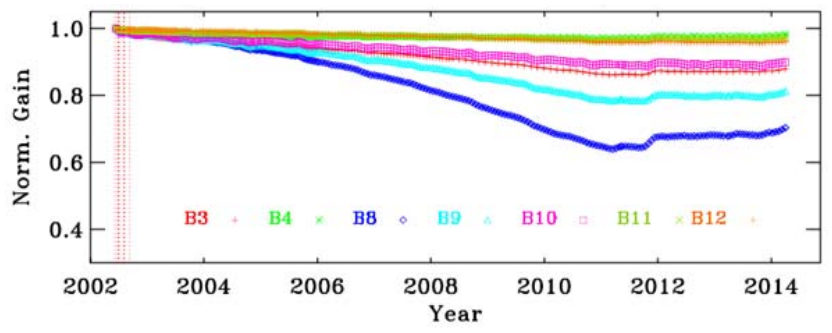

Figure 7 Normalized gains of Aqua MODIS VIS bands

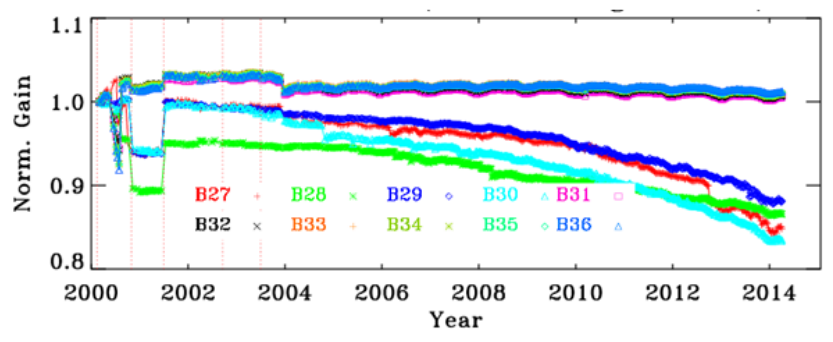

Figure 8 Normalized gains of Terra MODIS LWIR bands

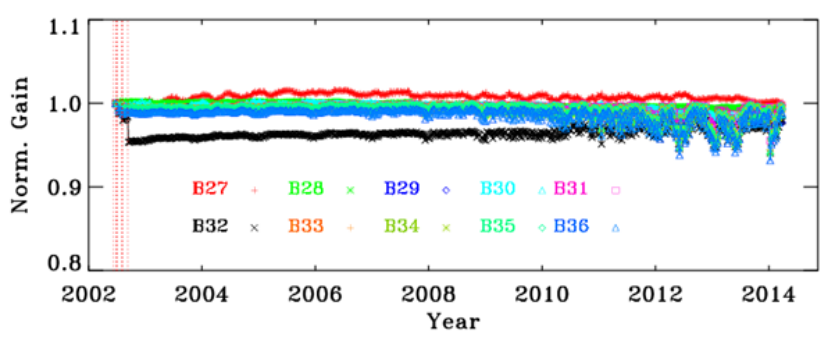

Figure 9 Normalized gains of Aqua MODIS LWIR bands

The TEB performance is characterized using the reciprocal of the linear calibration term b1 (also referred to as the instrument gain). Figures 8 and 9 show the time series of response changes (normalized to the first point) for the LWIR bands of Terra and Aqua MODIS respectively. The fluctuations in the gain for Aqua LWIR bands in recent years are attributed to the slow decrease in the CFPA cooler margin. Over the lifetime of both MODIS instruments the gain for the MWIR bands has varied within $\pm 1 \%$. In contrast, the long term trending of the gain of Terra LWIR PV/PC bands indicate noticeable changes; have drifted by about $10 \%$ for the PV and by about $2 \%$ for the PC LWIR bands respectively.

The on-orbit changes in the spectral characteristics, such as detector center wavelengths (CW) and bandwidths (BW), for the VIS and NIR spectral bands, are monitored by the SRCA when operated in its spectral mode. The on-orbit change in the bandwidths is relatively small for both instruments, less than $1 \mathrm{~nm}$ for most bands. The changes in the center-wavelengths have been less than $0.5 \mathrm{~nm}$ for most bands. Bands designed with broader bandwidths, such as bands 1 and 19 (50 nm BW) have experienced slightly larger shifts in CW and BW. Due to several lamp failures during the first few years of each mission, the SRCA spectral mode is no longer able to run a $30 \mathrm{~W}$ calibration. In its place, a modified, $20 \mathrm{~W}$ calibration was applied and continues to be in operation. The SRCA spatial mode is used to track the sensor spatial characteristics such as band-toband registration (BBR) in scan and track directions. In the case of Terra MODIS, only a few bands fail to meet the specification of $\pm 0.1 \mathrm{~km}$. Results from Aqua MODIS show large BBR offsets (up to $0.4 \mathrm{~km}$ ) for the cold FPA (SMIR and LWIR) bands relative to the warm FPA (VIS and NIR) bands. This problem was identified prelaunch and has remained the same on-orbit.

\section{FUTURE IMPROVEMENTS}

There have been a number of changes and improvements made in the latest L1B collection 6 (C6), including use of a consistent calibration and data quality assurance (QA) Look-Up Table (LUT), implementation of new algorithms to reduce the calibration impact due to variations in Aqua CFPA temperatures, and an improved approach to characterize the RSB RVS [5]. However, accurately tracking on-orbit changes in sensor RVS remains a major challenge. In C6, observations at different AOI over selected ground targets are used to derive RVS, especially for the bands with large differences between their results based on SD and lunar observations. During the initial release of C6, the EV-based RVS approach was only applied to a few selected VIS bands (Terra bands 1-4, 8 and 9 and Aqua bands 8 and 9). An extension of the same algorithm has now been implemented for Terra MODIS band 10 and is being considered for other VIS/NIR bands.

Large mirror (optics) degradation will not only impact sensor RVS but also change the sensor's polarization sensitivity, which is also a function of wavelengths and AOI. In order to accurately track changes in wavelength, AOI, and mirror side dependent polarization properties, more effort is needed to develop and implement different 
strategies, including using targets with different polarization properties. Currently, MCST is working closely with science groups in support of their study and implementation of polarization corrections for a number of Terra MODIS C6 data products. Future improvements of MODIS RSB RVS characterization algorithm are also being planned and examined.

MODIS SDSM detectors cover wavelengths from 0.41 to $0.94 \mu \mathrm{m}$ and the SD degradation is much slower at longer wavelengths. As a result, no correction of SD degradation is applied for the SWIR bands. After more than 14 years of onorbit operation, however, Terra MODIS SD degradation at $0.936 \mu \mathrm{m}$, as measured by the SDSM detector 9, has reached $2.3 \%$. The earth-view observations over pseudoinvariant ground targets revealed a long-term upward drift for band 5 of Terra MODIS. This upward drift is likely due to the degradation of the $\mathrm{SD}$, unaccounted for in the gain computation. Consequently, an EV-based correction has been formulated and will be applied to the band 5 gain coefficients. The drift for other SWIR bands is currently within $0.3 \%$; however a careful monitoring of this change is vital to maintain the accuracy of the SWIR band calibration. Currently, the SD degradation at $0.94 \mu \mathrm{m}$ is about $0.6 \%$ for Aqua MODIS and its calibration impact is expected to be much smaller than Terra MODIS. Since VIIRS has the same SD and SDSM system for its RSB calibration, similar SD degradation impact analysis and correction strategy should be considered for the SWIR bands. Currently, the VIIRS SD degradation has exceeded the Aqua MODIS.

For a few VIS bands, the detector responses have significantly decreased and, at the same time, the calibration target (SD) has shown signs of significant degradation at their wavelengths. Because of this, the current RSB calibration algorithm, based on a simple linear ratio approach, needs to be carefully examined to account for potential nonlinear effects.

In the case of MODIS TEB several improvements have been applied in C6. One of the major improvements is the removal (or deduction) of a cold scene bias as observed between MODIS and AIRS measurements [6]. For Terra MODIS, the photovoltaic (PV) LWIR bands have tended to show various degrees of noisy responses since 2010, which have manifested as striping artifacts in the EV imagery. Recent investigations using on-orbit lunar measurements show that the PV LWIR bands have been significantly affected by the electronic crosstalk. In support of future calibration improvement, a linear characterization and correction algorithm has been applied to band 27 responses
[7]. Similar effort is also being applied to other PV LWIR bands.

\section{ACKNOWLEDGEMENTS}

Authors would like to acknowledge the continuing support provided by the MODIS science discipline representatives and assistance from other MCST members.

\section{REFERENCES}

[1] V. Salomonson, W. Barnes, X. Xiong, S. Kempler, and E. Masuoka, "An Overview of the Earth Observing System MODIS Instrument and Associated Data Systems Performance," IEEE International Geoscience and Remote Sensing Symposium (IGARSS), doi:10.1109/IGARSS.2002.01025746, pp. 970-972, 2002.

[2] X. Xiong, B. Guenther, A. Angal, W. Barnes, V. Salomonson, J. Sun, and B. Wenny, "MODIS radiometric calibration program, methods and results," IEEE International Geoscience and Remote Sensing Symposium, doi: 10.1109/IGARSS.2012.6352570, pp. 6924-6927, 2012.

[3] Xiong X, K. Chiang, J. Esposito, B. Guenther and W.L. Barnes, "MODIS On-orbit Calibration and Characterization," Metrologia 40, 89-92, 2003.

[4] Xiong, X., J. Butler, N. Lei, J. Sun, J. Fulbright, Z. Wang, J. Mcintire, and A. Angal, "Improvements of VIIRS and MODIS solar diffuser and lunar calibration," Proc. SPIE 8866, Earth Observing Systems XVIII, 88661M, 2013.

[5] Toller, G., X. Xiong, J. Sun, B. N. Wenny, X. Geng, J. Kuyper, A. Angal, H. Chen, S. Madhavan, and A. Wu, "Terra and Aqua Moderate-resolution Imaging Spectroradiometer Collection 6 Level 1B Algorithm," Journal of Applied Remote Sensing, vol. 7, issue 1, 2013.

[6] Wenny, B. N., X. Xiong, and S. Madhavan, "Evaluation of Terra and Aqua MODIS thermal emissive band calibration consistency," Proc. SPIE 8533, Sensors, Systems, and Next-Generation Satellites XVI, 853317, 2012.

[7] Sun, J., X. Xiong, S. Madhavan, and B. N. Wenny, "Terra MODIS Band 27 Electronic Crosstalk Effect and Its Removal," IEEE Transactions on Geoscience and Remote Sensing, vol. 52, issue 3, pp. 1551-1561, 2014. 